\title{
Striatal interneurons in dissociated cell culture
}

\author{
S. C. Schock · K. S. Jolin-Dahel • P. C. Schock • \\ W. A. Staines $\cdot$ M. Garcia-Munoz • \\ Gordon W. Arbuthnott
}

Accepted: 4 May 2010 / Published online: 19 May 2010

(C) Springer-Verlag 2010

\begin{abstract}
In addition to the well-characterized direct and indirect projection neurons there are four major interneuron types in the striatum. Three contain GABA and either parvalbumin, calretinin or NOS/NPY/somatostatin. The fourth is cholinergic. It might be assumed that dissociated cell cultures of striatum (typically from embryonic day E18.5 in rat and E14.5 for mouse) contain each of these neuronal types. However, in dissociated rat striatal (caudate/putamen, $\mathrm{CPu}$ ) cultures arguably the most important interneuron, the giant aspiny cholinergic neuron, is not present. When dissociated striatal neurons from E14.5 Sprague-Dawley rats were mixed with those from E18.5 rats, combined cultures from these two gestational periods yielded surviving cholinergic interneurons and representative populations of the other interneuron types at 5 weeks in vitro. Neurons from E12.5 CD-1 mice were combined with $\mathrm{CPu}$ neurons from E14.5 mice and the characteristics of striatal interneurons after 5 weeks in vitro were determined. All four major classes of interneurons were identified in these cultures as well as rare tyrosine hydroxylase positive interneurons. However, E14.5 mouse $\mathrm{CPu}$ cultures contained relatively few cholinergic interneurons rather than the nearly total absence seen
\end{abstract}

S. C. Schock · K. S. Jolin-Dahel · P. C. Schock · W. A. Staines Department of Cellular and Molecular Medicine,

University of Ottawa, Ontario, Canada

M. Garcia-Munoz · G. W. Arbuthnott ( $\square)$

Brain Mechanisms for Behaviour Unit,

Okinawa Institute of Science and Technology Promotion

Corporation, 1919-1 Tancha, Onna-son,

Kunigami-gun, Okinawa 904-0412, Japan

e-mail: Gordon@ oist.jp in the rat. A later dissection day (E16.5) was required to obtain mouse $\mathrm{CPu}$ cultures totally lacking the cholinergic interneuron. We show that these cultures generated from two gestational age cells have much more nearly normal proportions of interneurons than the more common organotypic cultures of striatum. Interneurons are generated from both ages of embryos except for the cholinergic interneurons that originate from the medial ganglionic eminence of younger embryos. Study of these cultures should more accurately reflect neuronal processing as it occurs in the striatum in vivo. Furthermore, these results reveal a procedure for parallel culture of striatum and cholinergic depleted striatum that can be used to examine the function of the cholinergic interneuron in striatal networks.

Keywords Striatum - Cholinergic interneurons · Cell culture $\cdot$ Basal ganglia
Abbreviations
AChE Acetylcholinesterase
CalR Calretinin
ChAT Choline acetyltransferase
$\mathrm{CPu} \quad$ Caudate/putamen
DARPP Dopamine- and cyclic AMP-regulated phosphoprotein
EdU 5-Ethynyl-2'-deoxyuridine
GABA Gamma-aminobutyric acid
GAD Glutamic acid decarboxylase
GFAP Glial fibrillary protein
GFP Green fluorescent protein
HBSS Hank's balanced salt solution
LGE Lateral ganglionic eminence
L-glut L-Glutamine
MGE Medial ganglionic eminence
NeuN Neuronal neuclei 


$\begin{array}{ll}\text { NGF } & \text { Nerve growth factor } \\ \text { NOS } & \text { Nitric oxide synthase } \\ \text { NPY } & \text { Neuropeptide Y } \\ \text { ParV } & \text { Parvalbumin } \\ \text { PBS } & \text { Phosphate buffered saline } \\ \text { Pen/Strep } & \text { Penicillin/streptomycin } \\ \text { PNBM } & \text { Primary neuron basal medium } \\ \text { PNGM } & \text { Primary neuronal growth medium } \\ \text { SOM } & \text { Somatostatin } \\ \text { TANs } & \text { Tonically active neurons } \\ \text { TH } & \text { Tyrosine hydroxylase } \\ \text { vAChT } & \text { Vesicular acetylcholine transporter } \\ \text { vGAT } & \text { Vesicular GABAergic transporter } \\ \text { vGLUT } & \text { Vesicular glutamatergic transporter }\end{array}$

\section{Introduction}

The largest nucleus of the basal ganglia, the striatum-caudate/putamen $(\mathrm{CPu})$, is implicated in the integration of sensorimotor, cognitive and motivational information arriving from its inputs, of which the most well-studied have been the glutamatergic afferents from the whole cortical mantle and the dopaminergic fibers from substantia nigra. The integration of the information received is performed within the internal striatal networks. These networks are formed by medium spiny cells and four classes of interneurons. The interneurons involved in the regulation of $\mathrm{CPu}$ function are classified into four types based on their neurochemical profiles: choline acetyltransferase (ChAT), GABA and parvalbumin (ParV), GABA and calretinin (CalR), and GABA, somatostatin (SOM), neuronal nitric oxide synthase (NOS) and neuropeptide Y (NPY) (Kawaguchi et al. 1995; Kubota and Kawaguchi 1995; Tepper and Bolam 2004). In addition, in dopamine-deprived conditions, a tyrosine hydroxylase $(\mathrm{TH})$ positive striatal interneuron is observed (Ostergaard et al. 1991; Mura et al. 2000; Lopez-Real et al. 2003; Huot and Parent 2007).

Interneuron physiology

The largest striatal interneuronal cells have thick, relatively straight primary dendrites and have been characterized as cholinergic (Kawaguchi 1992; Bennett and Wilson 1999). The spontaneous regular activity of cholinergic interneurons is most likely produced by a self depolarizing mechanism. Indeed, in vivo they are recognized as tonically active neurons (TANs) (Bennett and Wilson 1999). Tonically active cholinergic interneurons respond together by ceasing to fire in a temporally coordinated fashion following stimuli associated with primary rewards (Sardo et al. 2000). Furthermore, cholinergic interneurons inhibit the glutamatergic input to medium spiny cells (Pakhotin and Bracci 2007).
The most numerous class of striatal interneurons were first described as GABA accumulating cells (Bolam et al. 1985). They display intense labeling to GABA and its synthesizing enzyme - glutamic acid decarboxylase (GAD) particularly the mRNA isoform $\mathrm{GAD}_{67}$ (Salin and Chesselet 1993). These interneurons express the calcium-binding protein parvalbumin (Gerfen et al. 1985; Cowan et al. 1990; Plotkin et al. 2005). Striatal interneurons identified by these markers are capable of firing at high rates with little adaptation and have been named fast spiking cells (Kawaguchi 1993). These interneurons receive a strong cortical glutamatergic input (Ramanathan et al. 2002) which activates primarily mGluR1 (Pisani et al. 2001). Fast spiking interneurons exert a strong inhibitory influence on CPu output neurons (Koos et al. 2004; Tepper et al. 2004; Mallet et al. 2005). They have been shown to be electrotonically coupled in local networks (Fukuda 2009).

The other interneurons, in comparison, produce longer lasting action potentials and after hyperpolarizations (Kawaguchi 1993; Partridge et al. 2009). The NOS-positive interneurons display low threshold calcium spikes and provide a GABA-mediated inhibitory control of $\mathrm{CPu}$ output neurons (Koos et al. 2004; Tepper and Bolam 2004; Partridge et al. 2009). Little is known about the electrophysiological properties of the calretinin-containing interneuron.

\section{Reasons for the study}

As techniques for culturing dissociated embryonic neurons progress, there is a need to develop methods to ascertain how well these cultures mirror the brain area from which they are derived. The best examples of this approach are probably to be found in the literature on explant cultures where small regions of embryonic tissue are removed and cultured. In some cases, explants from other connected areas of the brain are also added. These, and the slice cultures from which they derive, were originally designed for the study of the hippocampus (Gahwiler and Hefti 1985; Ostergaard et al. 1991). When studied quantitatively these cultures are shown to contain excess of interneurons (Ostergaard et al. 1995).

In contrast, during early experiments on the electrophysiological properties of neurons in conventional dissociated $\mathrm{CPu}$ cultures we noticed that there were no large cholinergic interneurons. This critical deficiency led us to assess the prevalence of $\mathrm{CPu}$ interneurons in dissociated cultures derived from different embryonic ages. In the following paper, we describe an optimized protocol for dissociated cultures of $\mathrm{CPu}$ which restores the cholinergic interneuron. In this way, striatal cultures can be studied in the context of the full compliment of interneurons and projection neurons in the proportions found in vivo. 


\section{Experimental procedures}

\section{Cryopreserved cell cultures}

Vials of mouse and rat primary neurons (QBM Cell Science, Ottawa, Canada) were stored in liquid nitrogen. Rat cells were thawed for $2.5 \mathrm{~min}$ at $37^{\circ} \mathrm{C}$ and resuspended in Neurobasal (Invitrogen) supplemented with B27 (2\% final volume, Invitrogen), $100 \mathrm{units} / \mathrm{ml}$ of penicillin, $100 \mu \mathrm{g} / \mathrm{ml}$ of streptomycin (pen/strep), L-glutamine (L-glut $2 \mathrm{mM}$ ) and nerve growth factor (NGF $100 \mathrm{ng} / \mathrm{ml}$ ). After thawing for $2.5 \mathrm{~min}$ at $37^{\circ} \mathrm{C}$, the mouse cells were suspended in prewarmed primary neuron basal medium (PNBM, Lonza, Walkersville, MD) and primary neuronal growth medium (PNGM, SingleQuots Lonza) supplemented with NGF $(100 \mathrm{ng} / \mathrm{ml})$. Rat and mouse cells were plated at a density of $1 \times 10^{5}$ cells/well on 96-well culture dishes coated with poly-D-lysine (BD BioCoat cellware, VWR). Cells were left in a humidified atmosphere $\left(5 \% \mathrm{CO}_{2} / 95 \%\right.$ air $)$ at $37^{\circ} \mathrm{C}$ for 5 weeks replacing $60 \%$ by volume of the media every 3-4 days, taking care to replace water lost by evaporation (Potter and DeMarse 2001) by adding $160 \mu \mathrm{l}$ sterile water per $1 \mathrm{ml}$ media to be added. No mitotic inhibitors were used.

Freshly dissociated cell cultures

Cultures of $\mathrm{CPu}$ neurons were prepared from embryonic mice (CD1 strain, Charles River) at either gestational day 12.5 or 14.5 or Sprague-Dawley rats at either gestational day 14.5 or 18.5. Dissection procedures were approved by the University of Ottawa Animal Care Committee. From each embryo, the CPu primordia MGE/LGE (medial/lateral ganglionic eminence) were removed and placed into ice cold $1 \times$ Hank's balanced salt solution (HBSS, Sigma-Aldrich). The dissected $\mathrm{CPu}$ were centrifuged at $1,000 \mathrm{rpm}$ for $5 \mathrm{~min}$ and the HBSS was aspirated off. Cells were mechanically dissociated in the presence of DNAse (200 units $/ \mathrm{ml}$, Sigma-Aldrich) at room temperature. Remaining tissue was left to settle for $2 \mathrm{~min}$ and the neurons in the supernatant were transferred to a new tube for estimating surviving cell numbers. Exclusion of Trypan blue (0.4\%, MP Biomedicals) was used to assess live cell number. The neurons were plated as stated above on poly-D-lysine-coated 96-well plates (BD BioCoat, VWR). For rat cells, Neurobasal medium supplemented with B27 (Invitrogen), pen/strep, L-glut and NGF $(100 \mathrm{ng} / \mathrm{ml})$ was used. Mouse cells were suspended in PNBM and PNGM SingleQuots (Lonza, Walkersville, MD) supplemented with NGF $(100 \mathrm{ng} / \mathrm{ml})$. Cells were plated as described above for cryopreserved cells. Again $60 \%$ of the media was replaced every 3-4 days, accounting for water loss. Cells were grown in a humidified atmosphere $\left(5 \% \mathrm{CO}_{2} / 95 \%\right.$ air) at $37^{\circ} \mathrm{C}$ for 5 weeks.
Green fluorescent protein mice

GFP mice were a kind gift from Dr. Douglas Gray. The generation of these mice has been described previously (Tsirigotis et al. 2001). The human UbiquitinC promoter was used to drive GFP expression in these mice which is not limited to any region or cell type within the brain.

EdU assay for cell birth dating

A click reaction 5-ethynyl-2'-deoxyuridine (EdU) imaging kit (Click-iT, Invitrogen) was employed as per the manufacturer's instructions to label dividing cells. Briefly, on the same day after plating, cells were incubated with $5 \mu \mathrm{M}$ EdU for $4 \mathrm{~h}$ and then the media was changed. This $4 \mathrm{~h}$ treatment was repeated everyday for 7 DIV prior to fixation in $4 \%$ paraformaldehyde containing $7 \%(\mathrm{v} / \mathrm{v})$ saturated picric acid for $45 \mathrm{~min}$ at room temperature. A $4 \mathrm{~h}$ treatment immediately following plating alone did not show any EdU-positive cells. Fixative was removed and the cells were washed twice in $10 \mathrm{mM}$ phosphate buffered saline (PBS). Wash solution was removed and $100 \mu \mathrm{l}$ of $0.5 \%$ Triton X-100 in PBS was added to each well for $20 \mathrm{~min}$ at room temperature. Following this, the buffer was removed and cells were washed twice in PBS. Then $50 \mu \mathrm{L}$ of Click-iT reaction cocktail was added to each well and incubated for $30 \mathrm{~min}$ at room temperature, protected from light. The reaction cocktail was removed and cells were washed once with PBS before they were immunostained with NeuN antibody to label all neurons in the well. See below for methods. Percentage of proliferating neurons present in each well was determined by counting the EdU positive cells compared to the NeuN positive cells.

Immunohistochemistry

Cultures were fixed in $4 \%$ paraformaldehyde containing $7 \%(\mathrm{v} / \mathrm{v})$ saturated picric acid for $45 \mathrm{~min}$ at room temperature. Cells were rinsed three times with $10 \mathrm{mM}$ PBS and stored at $4^{\circ} \mathrm{C}$ until assessed. Cultures were incubated with primary antibodies (see Table 1) diluted in $10 \mathrm{mM}$ PBS containing $0.3 \%$ Triton-X overnight at $4^{\circ} \mathrm{C}$, then rinsed with $10 \mathrm{mM}$ PBS for $5 \mathrm{~min}$. Appropriate secondary antibodies (Invitrogen) were used at a 1:400 dilution in $10 \mathrm{mM}$ PBS containing $0.3 \%$ Triton- $X$ and were incubated for $35 \mathrm{~min}$ at $37^{\circ} \mathrm{C}$, then rinsed again for $5 \mathrm{~min}$ prior to study using a Zeiss Axiovert 200M fluorescence microscope or a Zeiss Axioplan fluorescence microscope.

For double staining cells were incubated in primary antibody followed by secondary antibody as described above. Following positive cell staining, subsequent primary and secondary antibody incubation was performed on the same well. 
Table 1 Antibodies and sources

\begin{tabular}{llll}
\hline $\begin{array}{l}\text { Primary } \\
\text { Antibody }\end{array}$ & Species & Concentration & Supplier \\
\hline Calretinin & Goat & $1: 400$ & Swant, Bellinzona \\
Somatostatin & Mouse & $1: 200$ & Gift from Dr. Fryer \\
NOS & Rabbit & $1: 200$ & Santa Cruz \\
ParV & Mouse & $1: 4,000$ & Sigma \\
vAChT & Rabbit & $1: 1,000$ & Synaptic-Systems \\
TH & Mouse & $1: 50$ & Boehringer Mannheim \\
& & & Biochemica \\
AChE & Rabbit & $1: 1,000$ & Gift from \\
& & & Dr. J. Massoulie \\
vGAT & Rabbit & $1: 500$ & Alpha Diagnostics \\
vGAT & Guinea Pig & $1: 500$ & Synaptic-Systems \\
vGLUT & Guinea Pig & $1: 500$ & Millipore \\
DARPP & Mouse & $1: 100$ & Cell Signaling \\
DARPP & Mouse & $1: 1,000$ & Chemicon \\
GFP & Mouse & $1: 1,000$ & Abcam \\
GFP & Goat & $1: 1,000$ & Abcam \\
GFAP & Mouse & $1: 200$ & Chemicon \\
A60/NeuN & Mouse & $1: 10$ & Mouse Hybridoma \\
\hline
\end{tabular}

In order to visualize dendrites of the cholinergic interneurons, cultures were first labeled with vAChT antibody to identify positive cholinergic interneurons and label axons. The coordinates of positive cells in the well were recorded and the well was restained with $\mathrm{AChE}$ to increase visualization of the cell body and stain the dendrites. vAChT positive cells previously recorded were re-photographed.

\section{Counting and statistics}

To determine cell viability and percent of progenitor cells present in the cultures, cell counting was performed with the public domain ImageJ software (NIH Image). Cells were labeled with anit-NeuN and/or EdU and five $10 \times$ pictures at a resolution of 950 pixels $/ \mathrm{mm}$ within each well were taken to represent the whole area of the well using a Zeiss Axioplan fluorescence microscope. These pictures were opened in ImageJ and the successive processing steps were (1) linear adjustment of the threshold so that only the NeuN or EdU positive cells were highlighted, (2) analysis of particles with a pixel size of 20 pixels ${ }^{2}$-infinity. The results obtained were reliable as tested against several independent counts by hand of the same culture. For interneuron percentages, cells positive for each type of interneuron were counted by hand and compared to all neurons in the culture well as assessed by NeuN positive cell counts. Counts were made on two wells from each of three independent experiments. Statistical significance was determined by a one-way ANOVA performed on log-transformed data. The range is indicated by standard deviation.

\section{Results}

Characterization of rat mixed gestational age $\mathrm{CPu}$ cultures

In order to create a more complete rat $\mathrm{CPu}$ culture we engineered a new technique of mixed gestational dissections. These cultures were made possible through the cryopreservation services of QBM Cell Science, Ottawa. Results were compared to fresh cultures with no differences in cell viability, percentages of neuronal type or morphology observed. All the results reported were obtained with cryopreserved cells.

To obtain primary cholinergic interneurons to combine with dissociated cholinergic deficient $\mathrm{CPu}$ cells from E18.5, we took advantage of the abundant pre-migratory $\mathrm{CPu}$ cholinergic cell bodies present in the MGE at E14.5 by performing a $\mathrm{CPu}$ primordium (MGE/LGE) dissection and dissociation at this time point. Dissections at E18.5 provide dissociated cultures containing all other types of interneurons as well as the GABAergic projection neurons. A ratio of $75 \%$ E18.5 striatal cells to $25 \%$ E14.5 striatal cells was used as it approximated the percentage of cholinergic neurons in striatum in vivo (Table 2). We determined the prevalence of $\mathrm{CPu}$ interneurons at 35 days in vitro in both the single gestational and mixed gestational cultures by immunohistochemistry (Fig. 1). Examination of all culture conditions showed the presence of calretinin, parvalbumin and somatostatin interneurons. Cholinergic interneurons were only observed in mixed gestational and single E14.5 dissociated cultures. Examination of the mixed gestational

Table 2 Proportions of interneurons in rat cultures

\begin{tabular}{lllll}
\hline$\%$ & E18.5 & E14.5 & E18.5/14.5 & CPu in vivo \\
\hline NOS & $1.11 \pm 0.19$ & $0.24 \pm 0.01^{* *}$ & $0.93 \pm 0.18$ & 0.8 \\
ParV & $0.27 \pm 0.04$ & $0.18 \pm 0.04$ & $0.37 \pm 0.07$ & 0.7 \\
vAChT & $0.01 \pm 0.01$ & $0.22 \pm 0.04^{* *}$ & $0.08 \pm 0.03 * *$ & 0.3 \\
CalR & $0.96 \pm 0.28$ & $1.01 \pm 0.05$ & $0.68 \pm 0.23$ & 0.8
\end{tabular}

Percent number \pm standard deviation of immunohistochemically identified interneurons observed in $\mathrm{CPu}$ neuronal cultures from embryonic rats obtained from at different gestational ages. Percentages were obtained by comparing neurons positive for each interneuron marker to the total neuronal population as assessed by NeuN positive cell staining The final column shows percent numbers from rat $\mathrm{CPu}$ histological sections reported by Tepper and Bolam (2004)

** $P<0.01$ and $* P<0.05$ show statistical significance for dissection dates in comparison to the typical striatal dissection date (E18.5) for each interneuron 
Fig. 1 Interneurons in rat mixed gestational striatal cultures. Inverted images from immunostaining of interneurons showing NOS, parvalbumin (ParV), vAChT, calretinin (CalR), tyrosine hydroxylase (TH) and the spiny projection neuron marker DARPP-32 (DARPP) in rat mixed gestational striatal cultures. Cultures were 35 DIV, calibration bar $=50 \mu \mathrm{m}$
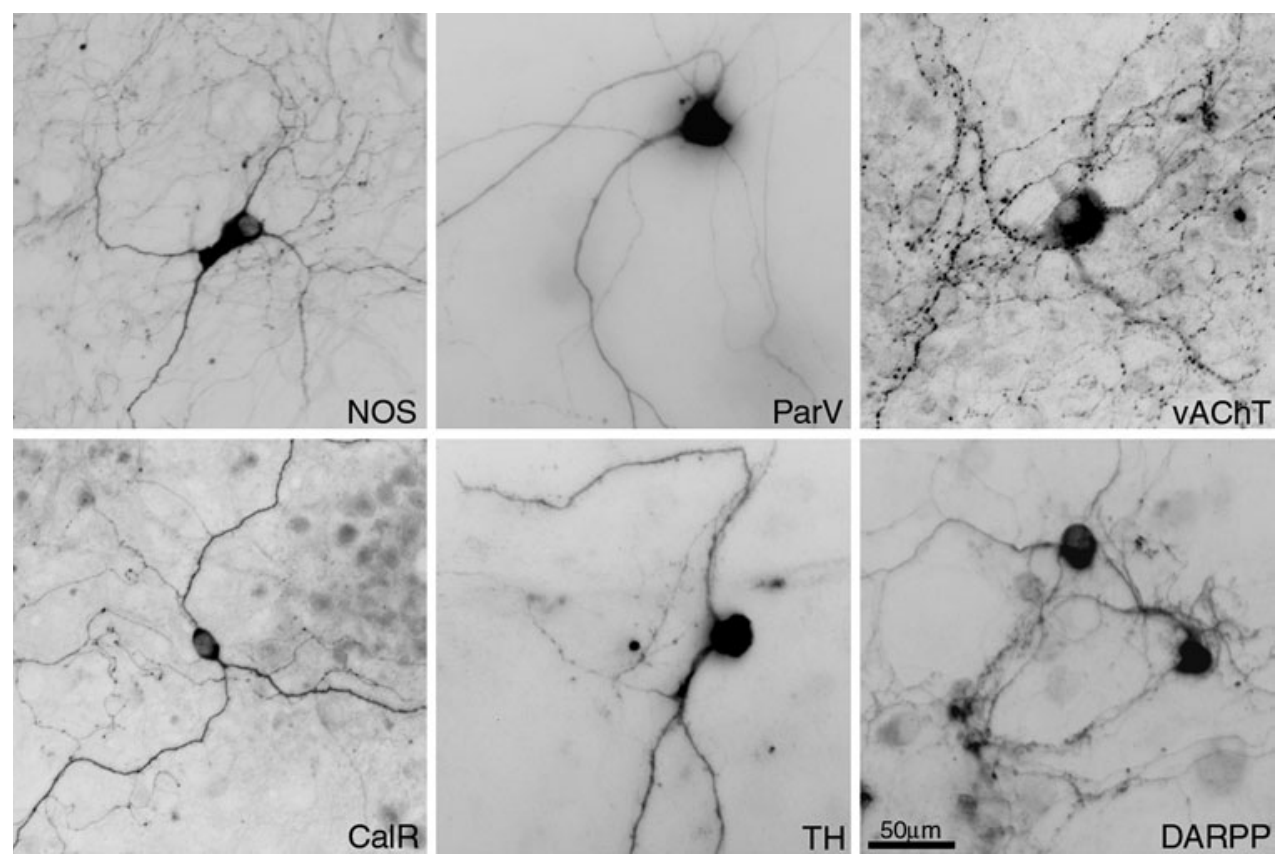

cultures showed the presence of all four types of interneurons at the percentages indicated in Table 2 which are in quite close accord with in vivo values (Tepper and Bolam 2004). The increase in cholinergic interneuron numbers in mixed gestational versus single E18.5 is nearly tenfold. Incidentally, we noted $\mathrm{TH}$ positive interneurons within both E18.5 single gestational cultures and mixed gestational cultures (Fig. 1).

The density of GABAergic terminals on each of the interneurons was approximated using immunohistochemistry for the vesicular GABAergic transporter (vGAT) co-labeled with markers for each of the interneurons. The parvalbumin and cholinergic interneurons have the highest amount of approximated vGAT terminals on their cell bodies and processes. NOS and calretinin interneurons showed almost no vGAT terminals and most of these interneurons were completely without GABAergic terminal approximation (Fig. 2).

Presence of progenitor cells in early dissociated cultures

In the dissociated $\mathrm{CPu}$ culture obtained at the early dissection time point, E14.5, there are a host of other cells in addition to the cholinergic interneuron. Many of these are undoubtedly neuronal progenitor cells. In order to determine the size of the population of progenitor cells in single and mixed gestational cultures we performed EdU assays and counted EdU positive neurons (NeuNpositive cells). We found that there were $10.38 \pm 1.10 \%$ EdU positive neurons in single E18.5 cultures compared to $13.09 \pm 2.08 \%$ in the mixed gestational cultures.
E14.5 cultures had $22.01 \pm 2.13 \%$ EdU positive neurons. EdU positive glia were found in only mixed and E18.5 single gestational cultures. It was found that there is a significant difference $(P<0.01)$ between the E14.5 cultures and the E18.5 and mixed gestational cultures in regards to the quantity of progenitor cells present. However, there is no significant difference between the E18.5 and mixed gestational cultures in the quantity of progenitor cells $(P=0.072)$. This suggests that one theoretical drawback to our mixed gestational procedure, the addition of undifferentiated progenitor cells, is not a limitation. After the first week, the number of EdU positive cells present in the E14.5 cultures was reduced to $0.21 \pm 0.05 \%$, showing that the neuronal cell proliferation is occurring within the first week after plating.

\section{Cell death over time}

In order to determine the quality of our cultures over time, we performed cell counts on neurons immunopositive for $\mathrm{NeuN}$ at various weeks in vitro. Comparisons between weeks for each dissection type did not show a significant decline in cell number over time. For example, at week 1 the average number of cells in a culture well was $15,505.3 \pm 384.5$. At week 3 the average number of cells per well was $14,121.1 \pm 104.9$ cells compared to week 5 which had $13,756.0 \pm 352.4$ cells/well, still $89 \%$ of the original neuron numbers. Numbers of neurons in single versus mixed gestational $\mathrm{CPu}$ culture types are similar for all weeks. 

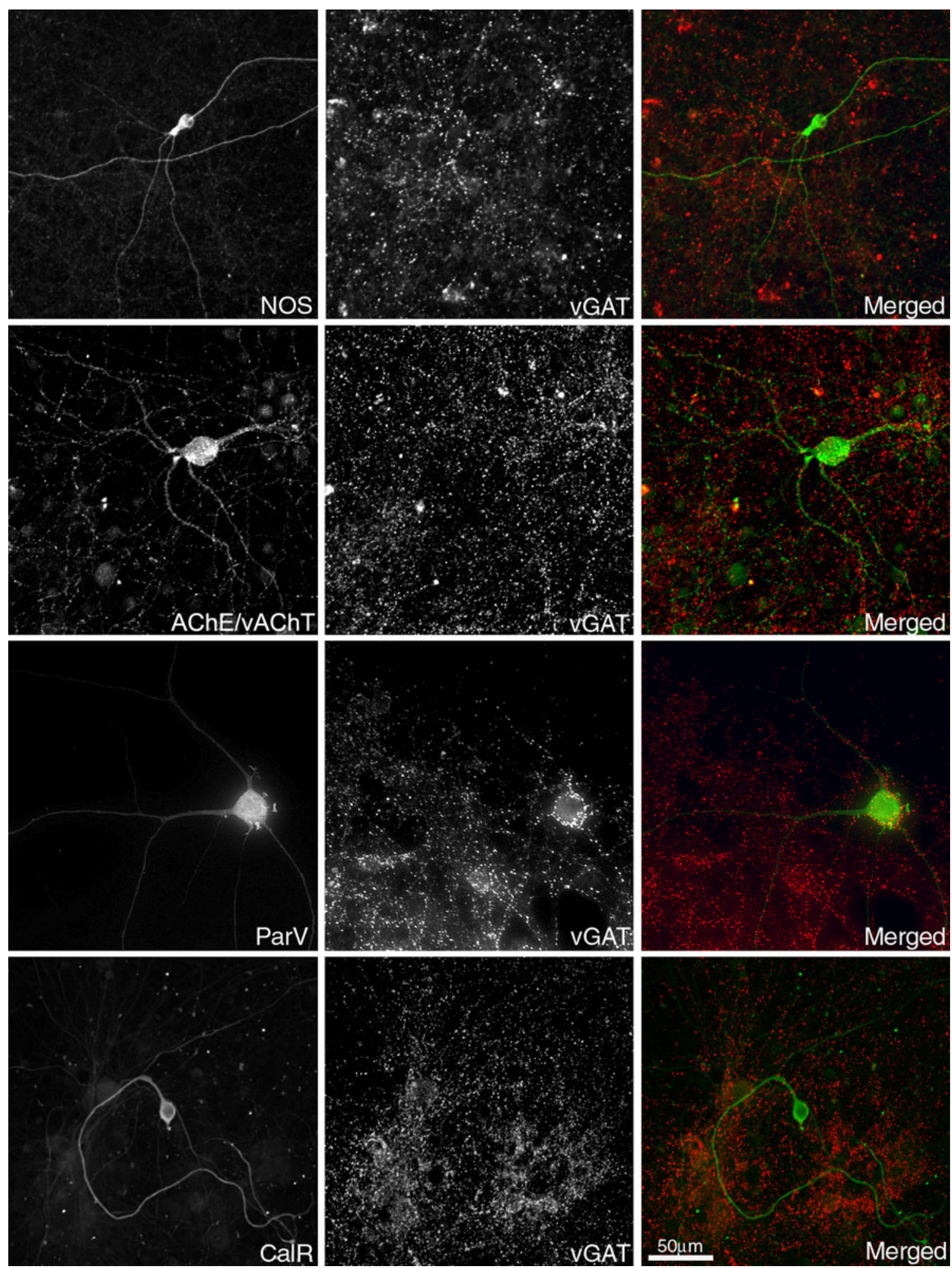

Fig. 2 GABAergic innervation of rat striatal interneurons in mixed gestational cultures. Two color immunohistochemical staining showing NOS, AChE/vAChT, parvalbumin (ParV), and calretinin (CalR) immunoreactive neurons in close approximation to vGAT positive terminals. Images are of $1 \mu \mathrm{M}$ single optical sections. Cultures were 35 DIV, calibration bar $=50 \mu \mathrm{m}$ 
Table 3 Proportions of interneurons in mouse cultures

\begin{tabular}{llllll}
\hline$\%)$ & E12.5 & E14.5 & E16.5 & E14.5/12.5 & CPu in vivo \\
\hline NOS & $1.77 \pm 0.13$ & $1.75 \pm 0.26$ & $1.71 \pm 0.15$ & $1.32 \pm 0.32$ & 0.8 \\
ParV & $0.35 \pm 0.08$ & $0.28 \pm 0.08$ & $0.29 \pm 0.06$ & $0.45 \pm 0.08^{*}$ & 0.7 \\
vAChT & $0.29 \pm 0.08^{* *}$ & $0.07 \pm 0.03$ & $0.01 \pm 0.05^{*}$ & $0.16 \pm 0.06^{*}$ & 0.3 \\
CalR & $1.52 \pm 0.21^{* *}$ & $0.78 \pm 0.31$ & $0.62 \pm 0.12$ & $1.25 \pm 0.18^{*}$ & 0.8 \\
\hline
\end{tabular}

Percent number \pm standard deviation of immunohistochemically identified interneurons observed in CPu neuronal cultures obtained from embryonic mice at different gestational ages. Percentages were obtained by comparing neurons positive for each interneuron marker to the total neuronal population as assessed by NeuN positive cell staining

For comparison the final column again shows percent numbers from rat CPu histological sections reported by Tepper and Bolam (2004)

$* * P<0.01$ and $* P<0.05$ show statistical significance for dissection dates in comparison to the typical striatal dissection date (E14.5) for each interneuron

Fig. 3 Interneurons in mouse mixed gestational striatal cultures. Inverted images of interneurons immunoreactive for NOS, parvalbumin (ParV), vAChT and calretinin (CalR) in mouse mixed gestational striatal cultures. Cultures were 35 DIV, calibration bar $=50 \mu \mathrm{m}$

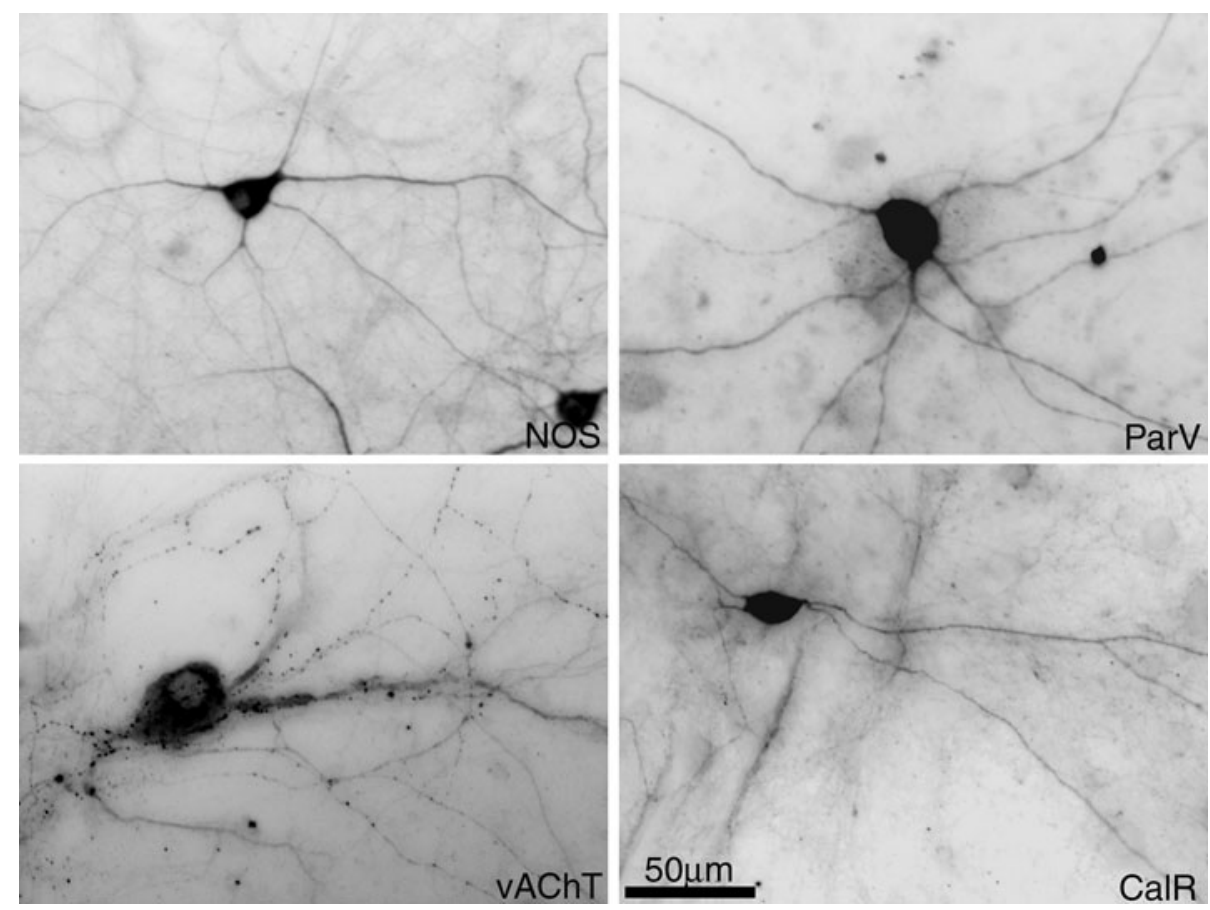

Characterization of mouse $\mathrm{CPu}$ cultures

Conventional mouse $\mathrm{CPu}$ dissections are performed at E14.5. This represents a late stage dissection analogous to E18.5 in the rat. Unlike in the rat, cholinergic neurons were present in dissociated late stage cultures, however, they were far less abundant than in vivo. After examining a number of earlier dissection dates in order to obtain the cholinergic interneurons, E12.5 was selected. This time point yielded four times as many cholinergic neurons (see Table 3). In the mixed gestational cultures (75\% E14.5 and $25 \%$ E12.5) all interneuron types were present (Fig. 3) and the percentage of interneurons was similar to that found in the mixed gestational rat cultures except that the NOS and calretinin interneurons were at a slightly higher percentage. The mixed gestational mouse cultures have two times as many cholinergic interneurons as single E14.5 cultures.
The date of origin of the striatal neurons in culture was determined by preparing mixed gestational cultures using E12.5 CPu obtained from GFP-transgenic mice. Immunostaining for interneuronal markers in addition to GFP confirmed that cholinergic interneurons were derived from the E12.5 tissue (i.e. they were GFP positive). The NOS, parvalbumin and calretinin interneurons, however, arose from both E14.5 and E12.5 CPu tissues (Fig. 4).

In order to obtain a greater yield of cholinergic interneurons, MGE dissections were compared to LGE alone or MGE/LGE dissections. Cholinergic interneurons were more prevalent in MGE dissections compared to LGE (Table 4). The cholinergic neurons from the MGE seem to be healthier, be higher in number and have a more extensive dendritic arborization than neurons obtained from the MGE/LGE dissections (Fig. 5). Similar observations were made for the parvalbumin interneuron. 

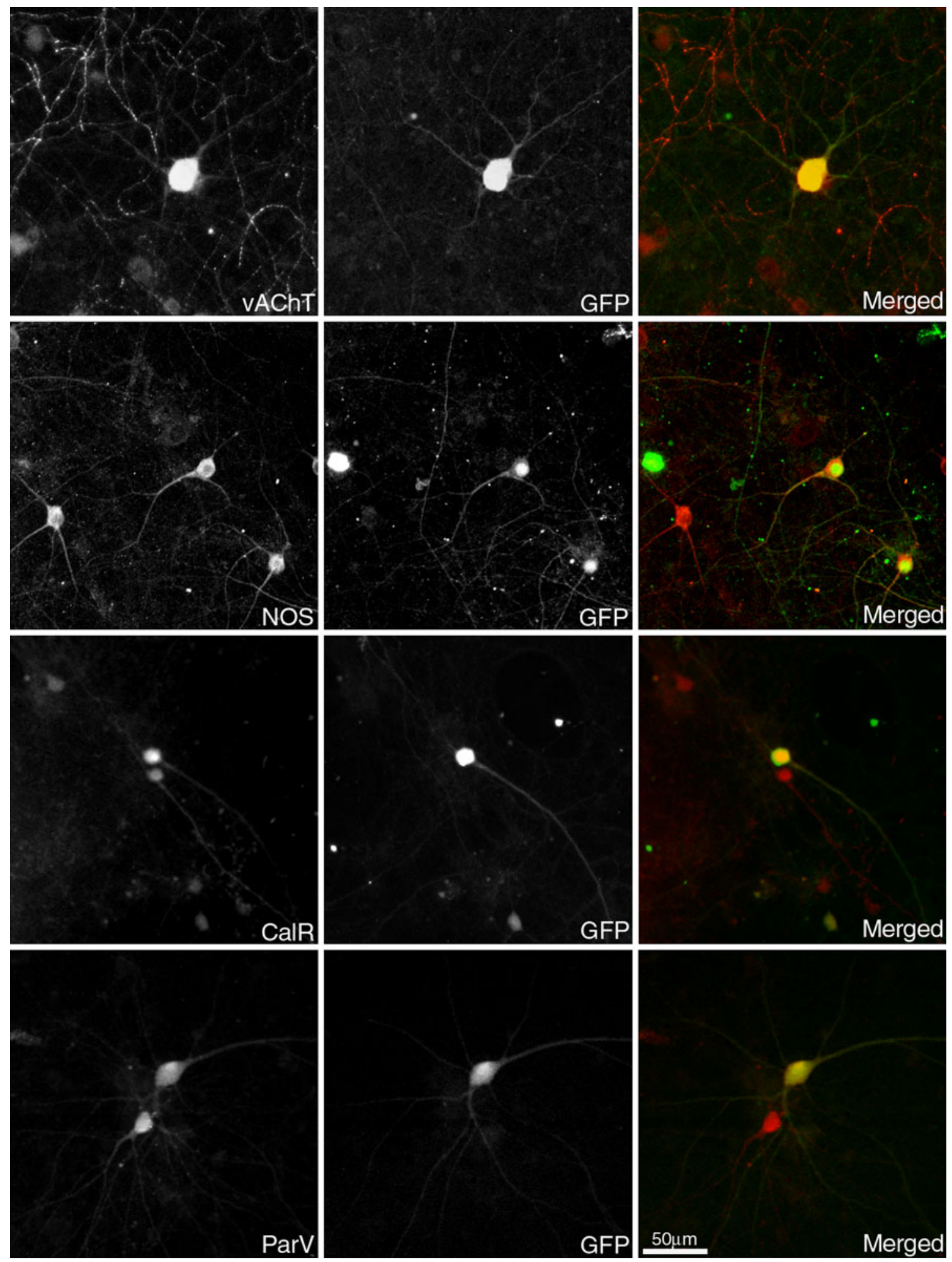

Fig. 4 Identification of the source of striatal interneurons in mouse mixed gestational cultures. GFP positive E12.5 striatum was cocultured with wildtype E14.5 striatum. All vAChT-positive cholinergic neurons had GFP fluorescence and thus were identified as having come

from the GFP positive E12.5 CPu. NOS, CalR and ParV are present in both the E12.5 and E14.5 CPu (are either GFP positive or GFP negative). Images are of $1 \mu \mathrm{M}$ single optical section. Cultures were $35 \mathrm{DIV}$, calibration bar $=50 \mu \mathrm{m}$ 
Table 4 The source of embryonic interneurons in the ganglionic eminence

\begin{tabular}{lllllrl}
\hline$\%)$ & E12.5 CPu & E12.5 MGE & E12.5 LGE & E14.5 CPu & E14.5 MGE & E14.5 LGE \\
\hline NOS & $1.72 \pm 0.09$ & $0.81 \pm 0.03^{*}$ & $0.75 \pm 0.26^{* *}$ & $1.81 \pm 0.61$ & $1.83 \pm 0.28$ & $1.65 \pm 0.28$ \\
ParV & $0.29 \pm 0.04$ & $0.56 \pm 0.05^{*}$ & $0.11 \pm 0.02^{* *}$ & $0.32 \pm 0.05$ & $0.21 \pm 0.01$ & $0.25 \pm 0.05$ \\
vAChT & $0.23 \pm 0.02^{* *}$ & $0.56 \pm 0.10^{* *}$ & $0.10 \pm 0.01$ & $0.08 \pm 0.03$ & $0.07 \pm 0.01$ & $0.06 \pm 0.02$ \\
CalR & $1.70 \pm 0.06^{*}$ & $0.96 \pm 0.05$ & $1.05 \pm 0.07$ & $1.02 \pm 0.27$ & $1.61 \pm 0.31 *$ & $1.36 \pm 0.33$ \\
\hline
\end{tabular}

Percent number \pm standard deviation of immunohistochemically identified interneurons observed in neuronal cultures obtained from embryonic mice at two gestational ages E12.5 or E14.5. Percentages were obtained by comparing neurons positive for each interneuron marker to the total neuronal population as assessed by NeuN positive cell staining. The numbers of interneurons observed in cultures obtained from $\mathrm{CPu}$ are compared to those observed in cultures from either the medial (MGE) or lateral ganglionic eminence (LGE)

** $P<0.01$ and $* P<0.05$ show statistical significance for dissection dates in comparison to the typical striatal dissection date (E14.5) for each interneuron
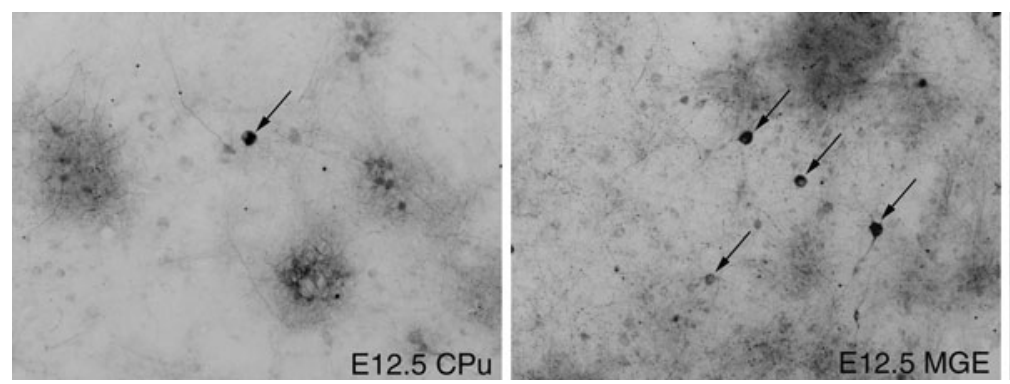

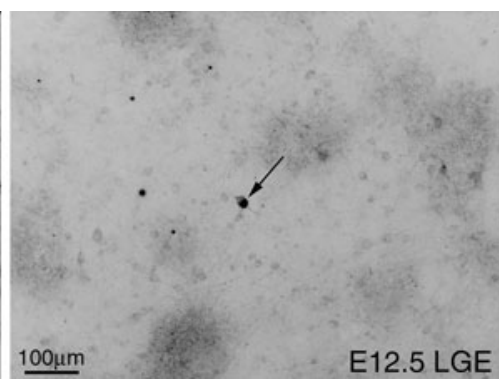

number of cell bodies as well as the axonal branching of the cholinergic interneuron in MGE cultures alone compared to either the $\mathrm{CPu}$ or LGE. Cultures were 35 DIV, calibration bar $=100 \mu \mathrm{m}$

represented. This expectation is likely incorrect with respect to many brain tissues due to the differential maturation of key cell types. This is certainly true in the case of dissociated cultures of striatum. Although most cell types are included in striatal cultures derived from the embryonic striatal primordium, such cultures lack arguably the most important striatal interneuron - the cholinergic interneuron.

This report contributes two important findings: (1) combining rat embryonic $\mathrm{CPu}$ neurons obtained from two gestational periods produces cultures containing cholinergic interneurons which had not been possible previously. (2) These cultures demonstrate interneuronal proportions similar to those calculated from histological section. The percentages of interneurons in the mixed gestational cultures are obtained from $75 \%$ late dissection neurons and $25 \%$ early dissection neurons. The only interneuron that is reduced in numbers in the mixed gestational cultures is the cholinergic interneuron, which is more concentrated at the early dissection time compared to the mixed gestational cultures. Even though all interneurons are represented in the early dissection date, the later dissection is still required to obtain a representative number of the other interneurons and the striatal projection neurons. It is widely known but poorly documented that dissociated rat $\mathrm{CPu}$ cultures (E18.5) do not contain cholinergic interneurons (personal communications). We confirmed this observation in our rat E18.5 cultures, where we failed to find significant numbers
A basic assumption of dissociated cultures of a brain region is that each of the important constituent neurons will be 
of cholinergic interneurons. This may be due to the fact that the ChAT positive interneurons have been post mitotic for 6.5 days while the other $\mathrm{CPu}$ interneurons have been post mitotic for only 2.5 days (Semba et al. 1988; Olsson et al. 1998). It is generally recognized that more mature neurons have a much lower survival rate in dissociated cultures. For the mouse striatal cultures, the typical dissociation date of E14.5 still contained cholinergic interneurons. One of the advantages to the preparation of these cultures is the ability to have a striatal network that is lacking cholinergic interneurons and one that contains them. Therefore, for the mouse cultures a later dissection date (E16.5) was identified as providing striatal cells devoid of cholinergic interneurons.

Many studies have characterized the use of rat striatum in organotypic cultures. Snyder-Keller and Costantini (1996) have described the development of CPu slices from embryonic rat brain and likewise found the interneuron populations to be present in those preparations. She has explored the signals for the development of the patch/ matrix pattern in her slice cultures (Snyder-Keller 1991; Snyder-Keller et al. 2001), and the influence of cortical innervation (Snyder-Keller 2004) and dopamine innervation (Snyder-Keller et al. 2008) upon it.

Using Gahwiler's method (Gahwiler 1984), Ostergaard cultured slices of striatum from neonatal rats and was able to demonstrate that the cholinergic innervation of the striatum by its interneurons developed seemingly normally (Ostergaard 1993). Also NPY, SOM, and NADPH diaphorase neurons were present in the cultures (Ostergaard et al. 1995). The major GABA interneurons were also present, at least in that a subset of the $\mathrm{CPu}$ neurons stained much more intensely for GAD and GABA (Ostergaard 1993). In quantitative studies, it seemed that the cultures had an increased proportion of interneurons (Ostergaard et al. 1995; West et al. 1996), perhaps because of their developmental age or perhaps because some of the output neurons were damaged by loss of their long axons (Lossi et al. 2009). In contrast, we see a smaller and more nearly normal percentage of all interneurons in our dissociated neuron cultures.

Interneurons in our mixed gestational cultures are present in proportions similar to in vivo percentages previously published (Kawaguchi et al. 1995; Centonze et al. 1999; Centonze et al. 2001; Tepper and Bolam 2004; Massouh et al. 2008). Even with the mixed age rat cultures the cholinergic neurons are less common than in vivo, but they are present. In the rat these interneurons are exclusively derived from the early dissection time point. At least they are absent in the absence of the cells derived from earlier embryos. In the mouse, we confirmed that the majority of the cholinergic neurons derive from earlier embryos by using GFP-tagged E12.5 CPu cells in our mixed gestational cultures. We therefore suggest that the earlier embryos are the source of the cholinergic neurons in the rat cocultures, rather than some factor derived from the earlier embryonic cells causes them to develop. In both mouse and rat, all the other interneurons develop from both early and later embryonic material.

Finally, we also observed TH positive interneurons in all three types of cultures in both rat and mouse models. The TH cells have been shown to be present in the striatum which is lacking dopaminergic input, such as in MPPinjected rats (O'Byrne et al. 2002) and MPTP-injected monkeys (Betarbet et al. 1997). Our studies suggest that the $\mathrm{TH}$ positive interneurons develop from embryonic $\mathrm{CPu}$ cells, and so are presumably present in normal as well as dopamine-depleted $\mathrm{CPu}$. Such a conclusion is also supported by their presence in slices from transgenic mice with GFP expressed on the TH promoter (Lopez-Real et al. 2003; Ibanez-Sandoval et al. 2007; Ibanez-Sandoval et al. 2008).

The ability to use cryopreserved neurons allowed us to easily produce mixed gestational age cultures and would also allow for coculture with substantia nigra, cortex or thalamus, brain areas which make connections with $\mathrm{CPu}$. When fresh cell isolations were compared to frozen cells, we saw no difference in the morphology, development, or viability over time of these neurons.

The precursors for the cholinergic interneurons in mice are born in the medial ganglionic eminence (MGE), migrate to the $\mathrm{CPu}$ at E13.5-14.5 and are amongst the first to enter the nucleus and develop projections (Marin et al. 2000). Therefore, they are well established by the conventional time point of neuronal dissociation (E14.5). The dissociation procedure presumably shears both axons and dendrites causing these cells to die during cell harvesting which results in few or no cholinergic interneurons in the final $\mathrm{CPu}$ cultures. However, the cholinergic neurons play a pivotal role in the overall function of the striatum and their presence is required to create a representative culture. For example, cholinergic interneurons provide an ongoing signal by firing tonically at approximately $5 \mathrm{~Hz}$ (Bennett and Wilson 1999; Bennett et al. 2000; Zhou et al. 2002) and oppose the effects of dopamine, another major regulator of the striatum. In this report, we show that with a novel procedure, which consisted of adding $\mathrm{CPu}$ neurons dissected from E12.5, containing the viable cholinergic neurons, to E14.5 mouse $\mathrm{CPu}$ culture, we were able to grow cholinergic interneurons. For rat cultures, adding $\mathrm{CPu}$ neurons from E14.5 to the E18.5 rat CPu cultures was equally successful in increasing the numbers of cholinergic interneurons.

One major application of our culture system is the ability to assess the contribution of the cholinergic interneuron to the overall functioning of the striatum. The role of the cholinergic interneuron in $\mathrm{CPu}$ neuronal network dynamics has been studied with pharmacological tools (Carrillo-Reid 
et al. 2009). Such examinations reinforce the importance of these neurons in $\mathrm{CPu}$ function and they may, like other neurons, be the source of neurotrophic factors whose pharmacological manipulation is less well known. We are now able to determine the role of the cells, rather than only their transmitter, by using late dissection cultures which are devoid of cholinergic neurons compared to the mixed gestational cultures containing the cholinergic neuron. Dissociated cultures will allow for the removal of the cholinergic interneuron from the striatal network in order to determine its function, a possibility that was not available when using previous methods of investigating the striatum, such as organotypic slice cultures.

Acknowledgments This work was funded in collaboration with OISTpc. We would like to thank Dr. Douglas Gray for the GFP mice used in this study.

\section{References}

Bennett BD, Wilson CJ (1999) Spontaneous activity of neostriatal cholinergic interneurons in vitro. J Neurosci 19:5586-5596

Bennett BD, Callaway JC, Wilson CJ (2000) Intrinsic membrane properties underlying spontaneous tonic firing in neostriatal cholinergic interneurons. J Neurosci 20:8493-8503

Betarbet R, Turner R, Chockkan V, DeLong MR, Allers KA, Walters J, Levey AI, Greenamyre JT (1997) Dopaminergic neurons intrinsic to the primate striatum. J Neurosci 17(17):6761-6768

Bolam JP, Powell JF, Wu JY, Smith AD (1985) Glutamate decarboxylase-immunoreactive structures in the rat neostriatum: a correlated light and electron microscopic study including a combination of Golgi impregnation with immunocytochemistry. J Comp Neurol 237:1-20

Carrillo-Reid L, Tecuapetla F, Ibanez-Sandoval O, Hernandez-Cruz A, Galarraga E, Bargas J (2009) Activation of the cholinergic system endows compositional properties to striatal cell assemblies. J Neurophysiol 101:737-749

Centonze D, Gubellini P, Bernardi G, Calabresi P (1999) Permissive role of interneurons in corticostriatal synaptic plasticity. Brain Res Brain Res Rev 31:1-5

Centonze D, Pisani A, Bonsi P, Giacomini P, Bernardi G, Calabresi P (2001) Stimulation of nitric oxide-cGMP pathway excites striatal cholinergic interneurons via protein kinase $G$ activation. J Neurosci 21:1393-1400

Cowan RL, Wilson CJ, Emson PC, Heizmann CW (1990) Parvalbumin-containing GABAergic interneurons in the rat neostriatum. J Comp Neurol 302:197-205

Fukuda T (2009) Network architecture of gap junction-coupled neuronal linkage in the striatum. J Neurosci 29:1235-1243

Gahwiler BH (1984) Slice cultures of cerebellar, hippocampal and hypothalamic tissue. Experientia 40:235-243

Gahwiler BH, Hefti F (1985) Striatal acetylcholinesterase-containing interneurons innervate hippocampal tissue in co-cultured slices. Brain Res 350:311-314

Gerfen CR, Baimbridge KG, Miller JJ (1985) The neostriatal mosaic: compartmental distribution of calcium-binding protein and parvalbumin in the basal ganglia of the rat and monkey. Proc Natl Acad Sci USA 82:8780-8784

Huot P, Parent A (2007) Dopaminergic neurons intrinsic to the striatum. J Neurochem 101:1441-1447
Ibanez-Sandoval O, Abercrombie ED, Koos T, Tepper JM (2007) Electrophysiology and morphology of a striatal dopaminergic neuron in mouse. Soc Neurosci Abstr 515:22

Ibanez-Sandoval O, Tecuapetla F, Shah F, Altinbilek B, Koos T, Tepper JM (2008) Synaptic connections of striatal dopaminergic neurons. Soc Neurosci Abstr 179:16

Kawaguchi Y (1992) Large aspiny cells in the matrix of the rat neostriatum in vitro: physiological identification, relation to the compartments and excitatory postsynaptic currents. J Neurophysiol 67:1669-1682

Kawaguchi Y (1993) Physiological, morphological, and histochemical characterization of three classes of interneurons in rat neostriatum. J Neurosci 13:4908-4923

Kawaguchi Y, Wilson CJ, Augood SJ, Emson PC (1995) Striatal interneurones: chemical, physiological and morphological characterization. Trends Neurosci 18:527-535

Koos T, Tepper JM, Wilson CJ (2004) Comparison of IPSCs evoked by spiny and fast-spiking neurons in the neostriatum. J Neurosci 24:7916-7922

Kubota Y, Kawaguchi Y (1995) Heterogeneity of neostriatal GABAergic interneurons. Basal Ganglia Society Meeting Abstracts 46

Lopez-Real A, Rodriguez-Pallares J, Guerra MJ, Labandeira-Garcia JL (2003) Localization and functional significance of striatal neurons immunoreactive to aromatic L-amino acid decarboxylase or tyrosine hydroxylase in rat Parkinsonian models. Brain Res 969:135-146

Lossi L, Alasia S, Salio C, Merighi A (2009) Cell death and proliferation in acute slices and organotypic cultures of mammalian CNS. Prog Neurobiol 88:221-245

Mallet N, Le Moine C, Charpier S, Gonon F (2005) Feedforward inhibition of projection neurons by fast-spiking GABA interneurons in the rat striatum in vivo. J Neurosci 25:3857-3869

Marin O, Anderson SA, Rubenstein JL (2000) Origin and molecular specification of striatal interneurons. J Neurosci 20:6063-6076

Massouh M, Wallman MJ, Pourcher E, Parent A (2008) The fate of the large striatal interneurons expressing calretinin in Huntington's disease. Neurosci Res 62:216-224

Mura A, Linder JC, Young SJ, Groves PM (2000) Striatal cells containing aromatic L-amino acid decarboxylase: an immunohistochemical comparison with other classes of striatal neurons. Neuroscience 98:501-511

O'Byrne MB, Bolam JP, Hanley JJ, Tipton KF (2002) Tyrosinehydroxylase immunoreactive cells in the rat striatum following treatment with MPP+. Adv Exp Med Biol 483:369-374

Olsson M, Bjorklund A, Campbell K (1998) Early specification of striatal projection neurons and interneuronal subtypes in the lateral and medial ganglionic eminence. Neuroscience 84:867-876

Ostergaard K (1993) Organotypic slice cultures of the rat striatum-I. A histochemical and immunocytochemical study of acetylcholinesterase, choline acetyltransferase, glutamate decarboxylase and GABA. Neuroscience 53:679-693

Ostergaard K, Schou JP, Gahwiler BH, Zimmer J (1991) Tyrosine hydroxylase immunoreactive neurons in organotypic slice cultures of the rat striatum and neocortex. Exp Brain Res 83:357-365

Ostergaard K, Finsen B, Zimmer J (1995) Organotypic slice cultures of the rat striatum: an immunocytochemical, histochemical and in situ hybridization study of somatostatin, neuropeptide $\mathrm{Y}$, nicotinamide adenine dinucleotide phosphate-diaphorase, and enkephalin. Exp Brain Res 103:70-84

Pakhotin P, Bracci E (2007) Cholinergic interneurons control the excitatory input to the striatum. J Neurosci 27:391-400

Partridge JG, Janssen MJ, Chou DY, Abe K, Zukowska Z, Vicini S (2009) Excitatory and inhibitory synapses in neuropeptide Y-expressing striatal interneurons. J Neurophysiol 102:3038-3045 
Pisani A, Bonsi P, Centonze D, Bernardi G, Calabresi P (2001) Functional coexpression of excitatory mGluR 1 and mGluR5 on striatal cholinergic interneurons. Neuropharmacology 40:460-463

Plotkin JL, Wu N, Chesselet MF, Levine MS (2005) Functional and molecular development of striatal fast-spiking GABAergic interneurons and their cortical inputs. Eur J Neurosci 22:1097-1108

Potter SM, DeMarse TB (2001) A new approach to neural cell culture for long-term studies. J Neurosci Methods 110:17-24

Ramanathan S, Hanley JJ, Deniau JM, Bolam JP (2002) Synaptic convergence of motor and somatosensory cortical afferents onto GABAergic interneurons in the rat striatum. J Neurosci 22:8158-8169

Salin P, Chesselet MF (1993) Expression of GAD (M(r) 67, 000) and its messenger RNA in basal ganglia and cerebral cortex after ischemic cortical lesions in rats. Exp Neurol 119:291-301

Sardo P, Ravel S, Legallet E, Apicella P (2000) Influence of the predicted time of stimuli eliciting movements on responses of tonically active neurons in the monkey striatum. Eur J Neurosci 12:1801-1816

Semba K, Vincent SR, Fibiger HC (1988) Different times of origin of choline acetyltransferase- and somatostatin-immunoreactive neurons in the rat striatum. J Neurosci 8:3937-3944

Snyder-Keller AM (1991) Development of striatal compartmentalization following pre- or postnatal dopamine depletion. J Neurosci 11:810-821

Snyder-Keller A (2004) Pattern of corticostriatal innervation in organotypic cocultures is dependent on the age of the cortical tissue. Exp Neurol 185:262-271
Snyder-Keller A, Costantini LC (1996) Glutamate receptor subtypes localize to patches in the developing striatum. Brain Res Dev Brain Res 94:246-250

Snyder-Keller A, Costantini LC, Graber DJ (2001) Development of striatal patch/matrix organization in organotypic co-cultures of perinatal striatum, cortex and substantia nigra. Neuroscience 103:97-109

Snyder-Keller A, Tseng KY, Lyng GD, Graber DJ, O'Donnell P (2008) Afferent influences on striatal development in organotypic cocultures. Synapse 62:487-500

Tepper JM, Bolam JP (2004) Functional diversity and specificity of neostriatal interneurons. Curr Opin Neurobiol 14:685-692

Tepper JM, Koos T, Wilson CJ (2004) GABAergic microcircuits in the neostriatum. Trends Neurosci 27:662-669

Tsirigotis M, Thurig S, Dube M, Vanderhyden BC, Zhang M, Gray DA (2001) Analysis of ubiquitination in vivo using a transgenic mouse model. Biotechniques 31:120-130

West MJ, Ostergaard K, Andreassen OA, Finsen B (1996) Estimation of the number of somatostatin neurons in the striatum: an in situ hybridization study using the optical fractionator method. J Comp Neurol 370:11-22

Zhou FM, Wilson CJ, Dani JA (2002) Cholinergic interneuron characteristics and nicotinic properties in the striatum. J Neurobiol 53:590-605 